\title{
Design Of Intelligent Home System Under Wifi Environment
}

\author{
Miao Yingkai \\ Henan University, Puyang Institute of Technology \\ Puyang, Henan 457000, China \\ 12101655@qq.com
}

Keywords: Smart Home, WiFi, CC3200

\begin{abstract}
The traditional intelligent home system is mostly ZigBee wireless technology network, with home gateway as the control center. The design of this essay takes the mobile phone as the control terminal, takes the $\mathrm{WiFi}$ as the communication means, removes the home gateway, carries on the long-distance control to the home equipment directly. Experiments show that the system is flexible and convenient to use, stable, easy to expand and upgrade, the user experience better.
\end{abstract}

\section{Introduction}

With the human communication technology and the development of Internet of Things technology, intelligent home is growing rapidly. More and more smart appliances into people's lives, so that people experience a more convenient, safe and efficient home life.

Traditional intelligent home system usually consists of three parts: home LAN, home gateway, external network. The home LAN connects home devices, intelligent switches, sensors, etc. to the ZigBee or other RF network to provide control signals and network information to the gateway. Home gateway is the core of the system, responsible for global control and communication with the external network functions. The user communicates with the gateway through the web page or the client and controls the home device. This intelligent home system after years of development, still not have a good application in the market, the following reasons:

1) ZigBee technology is not a unified standard, the development is more difficult, and with mobile phones and other smart devices are not compatible.

2) Must use the home gateway to work, the scope is limited.

3) Installation of network equipment is complex, the need for professional engineering staff.

4) Usually using web pages or computer client control, can not be used anytime, anywhere, the user experience is poor.

WiFi technology because of its high power, less network capacity in the field of intelligent home has not been widely used. But with the development of technology, many manufacturers introduced a low-power WiFi chip. At the same time, IEEE will release 802.11ah standards for Internet of things applications soon, the frequency of use of less than $1 \mathrm{GHz}$ license-free band. With greater coverage, support for more users, lower power consumption features, so WiFi in the smart home with a wider range of applications.

Nowadays, the penetration rate of smart phones is getting higher and higher, and the function is becoming more and more powerful. Along with the development of mobile phone $4 \mathrm{G}$ network, mobile phones have become the information center of people. Therefore, the smart phone as a smart home control center will be the development trend of home. 
In this paper, a common mobile phone as the control center to $\mathrm{WiFi}$ for home networking intelligent home system.

\section{The General Design}

The intelligent home system based on WiFi mainly includes the home appliance equipment control end and the mobile phone client end. User interaction through the mobile client, home appliance network equipment and control operations. Appliances directly from the wireless router access to the home network, by receiving from the mobile client network request, parsing out the control command, complete the corresponding control operation. The overall structure of the system diagram shown in Figure 1.

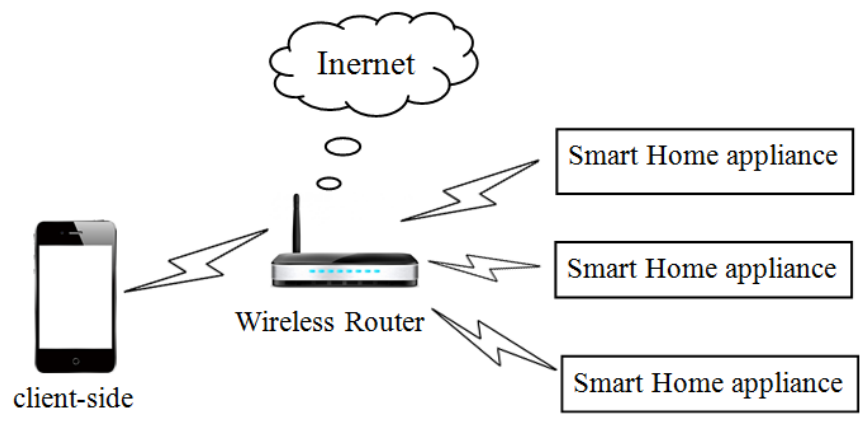

Figure 1 Smart home system diagram

\section{Intelligent Home Appliances Control Design}

The system of intelligent home appliances from the control end to receive mobile phone commands to control the appliance functions, the overall structure of the framework shown in Figure 2. The control-side core is a Texas Instruments (TI) CC3200 microcontroller based on the ARM Cortex-M4 core. CC3200 work at $80 \mathrm{MHz}$ frequency, with a fast parallel camera interface, I2S, SD / MMC, UART, SPI, I2C, ADC and other peripherals. The chip contains a dedicated $\mathrm{WiFi}$ module, can reduce the burden on the MCU, support 802.11.b / g / n RF and embedded TCP / IP, TLS / SS stack and a number of Internet protocols to support advanced low-power mode, Power saving demand. External serial flash memory to save the program and peripheral drivers, the system starts when the internal Flash boot loader (boot loader) to the external
Flash program loaded into the internal SRAM chip to run. Sensor module will be the external environment parameters passed to the control side for the MCU to deal with. The control end receives the mobile phone instruction, through the I / O mouth and the electrical appliances product connection and carries on the corresponding control, completes each kind of different function.

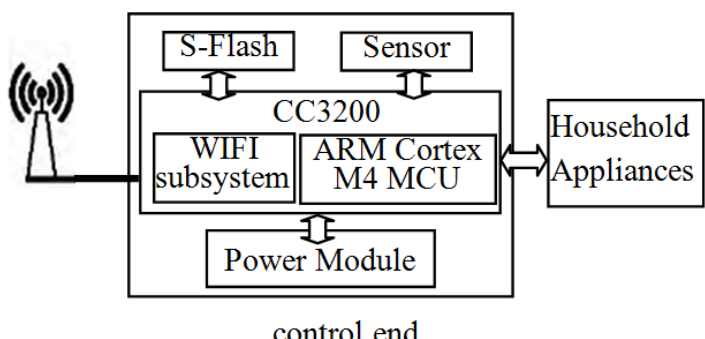

Figure 2 Control System Structure Diagram

Console software workflow shown in Figure 3. After powering on the system, grab the UDP packet to configure the network. If the configuration is not successful, enter the sleep mode to wait for the reset and restart the system. After the configuration is complete, Open the HTTP service, listen for HTTP requests, and perform the corresponding operations. 


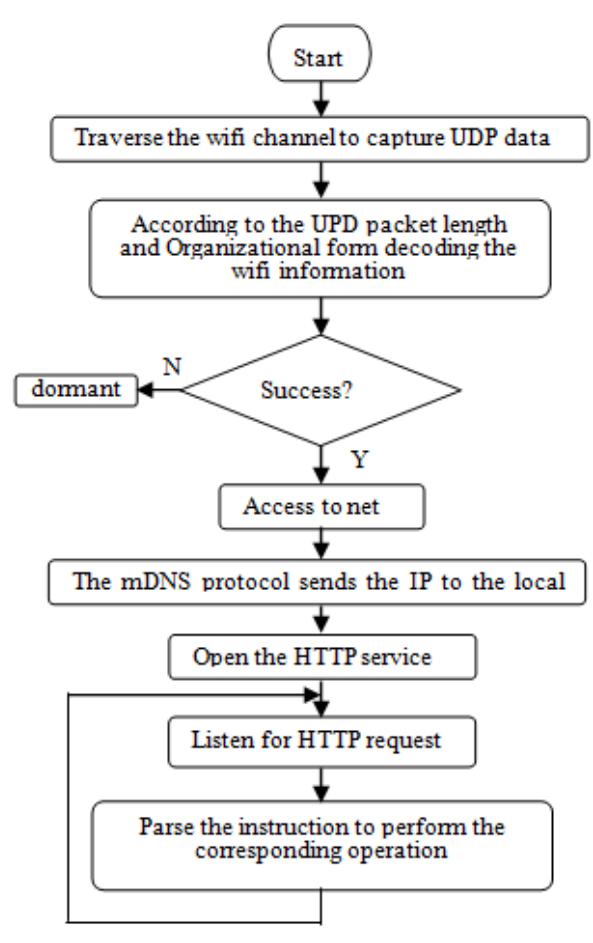

Figure3 Control side workflow

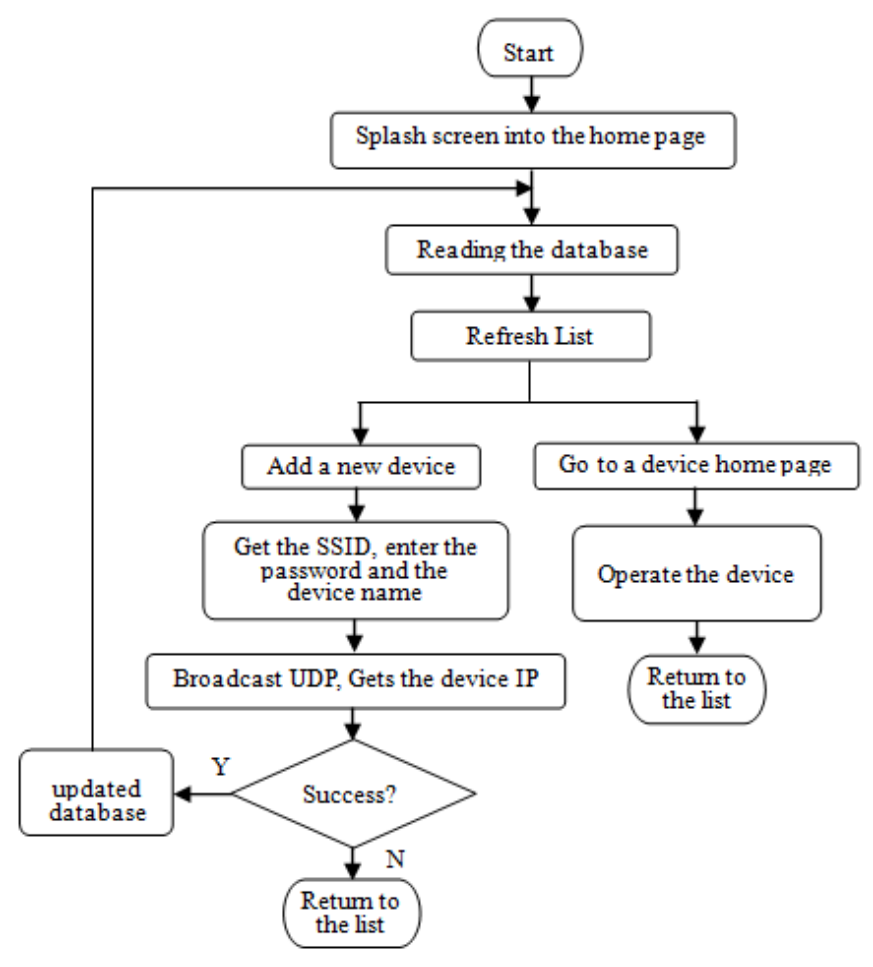

Figure 4 Mobile client workflow

\section{Network Communication}

\section{Mobile phone client software design}

Mobile phone client process can be divided into three modules: human-computer interaction module, network communication module and data processing module. Human-computer interaction module majors in transferring human's behavior into data, and then hand it to other modules, and reflects in UI screen to represent human's behavior; network communication module can build up relevant connection with smart home appliance and cloud servers, through the connection, the exchange of data can be achieved; data processing module can analyze and handle the data which is transferred from human-computer interaction module and network communication module.

The work flow of mobile phone client can be viewed on figure 4 .
The network communication in the system mainly includes two parts, one part is to configure the control end to insert the network, another part is the network communication between the mobile phone and the control end.

\section{A. Configuration Control Net}

Home appliances and mobile phones to communicate, first of all to access the home WiFi network. (2) mobile phones will be home router SSID and password sent to the hot spots; (3) WiFi devices Switch the operating mode to the terminal mode, use the received information to connect to the router in the home. This method of operation is complex, the phone needs to switch between different WiFi hotspots, the user experience is poor.

In this regard, The essay adopts a CC3200 based on a key network approach, the phone access to the home WiFi network, home appliances, power, the phone opens the client, the configuration page can automatically obtain the router SSID, the user simply enter the WiFi Password and custom 
device name, and then click the Configure button, you can automatically complete the device network, while access to the IP address of the appliance. The work process is divided into the following four steps:

1) After the control terminal power on, scan all WiFi hotspot channels, and then configure the chip to scan a channel to receive UDP packets, if not received UDP packets continue to configure the chip to scan in another channel. This loop until the receipt of UDP packets so far.

2) The mobile side encodes the wireless SSID and the password into the length byte of the UDP packet header, and repeats the broadcast of the UDP packet for $1 \mathrm{~min}$. At the same time, the Bonjour service based on the $\mathrm{mDNS}$ protocol is enabled to broadcast messages to the local network, who will provide the "local. _http, _tcp" service and wait for reply.

3) As the UDP packet frame format, the length of the packet is clearly visible, the control end receives UDP packets, according to the set of good coding, parsing out the corresponding information, access to the home $\mathrm{WiFi}$ network.

4) After the control end accesses the network, receives the UDP broadcast message, replies own equipment name and the IP address. The phone receives the reply message and processes it to complete the network configuration. 。

\section{B. UDP Broadcast Packets Encoding}

UDP protocol is a connectionless, unreliable transport layer protocol, the data packet format, including UDP headers and data. UDP data packet header is not encrypted can be intercepted by the source port, destination port, packet length and inspection and a total of four fields, each field is 2 bytes.
SSID and password will be converted into a series of delimiters, tag, high, low equivalent, and then sent out as the length of the packet.

Control to capture the UDP packet, read the packet length, according to the rules to identify, after identification by the received SSID and password information to $\log$ in the home WiFi network.

\section{Conclusion}

After the completion of the system design, the test can be achieved through the home network of home wireless control equipment, network equipment, convenient configuration, HTTP request is stable and reliable. Through the system can be centralized management of home appliances, home appliances to understand the current state of the latest operation.

The control system through the smart phone direct control of household equipment, eliminating the middle of the home gateway management, the use of $\mathrm{WiFi}$ transmission technology to better equipment compatibility.

As the mobile phone software and household equipment, the independence of the independence of hardware at the same time easy to use, easy to transplant and expansion, so that the system has a high market value.

\section{[1] AUST S, PRASAD R V , NIEMEGEERS I}

G. IEEE 802. 11ah: advantages in standards and further challenges for sub $1 \mathrm{GHz} \mathrm{Wi}-\mathrm{Fi}$

[C] .Communications( ICC) , 2012 IEEE International Conference on, 2012: 6885-6889.

[2] Texas Instruments Inc. CC3200 simplelink WiFi and IoT solution, a single chip wireless MCU

[EB/OL].(2014-06-01)

[2015-12-01] .http://www. ti. com. cn /cn/lit/ds/symlink/cc3200. pdf.

[3] TANENBAUM A S. Computing network (the fourth edition) $[\mathrm{M}]$. Pan Yueming, Translation. Beijing:

Tsinghua University Press,2004

[4] Dong Siqiao, Zhao Rongjian, Sun Tong. Design of Intelligent Home Control System Base on Wi Fi. Video Engineering. 2015.4: 89-90 
[5] Zhu Xinying, Chen Xi. The Design of Intelligent

Home Control System based on WIFI. Intelligent Computer and Applications. 2014.6: 80-82 